\title{
Detecting the Nonstationary Response of ENSO to Greenhouse Warming
}

\author{
A. TIMMERMANN
}

KNMI, De BiH, the Netherlands

(Manuscript received 8 January 1998, in final form 21 July 1998)

\section{ABSTRACT}

\begin{abstract}
On the basis of the latest greenhouse warming experiment performed with the Max-Planck Institut coupled atmosphere/isopycnal ocean model (ECHAM4/OPYC) it is shown that not only the climate mean but also the statistics of higher-order statistical moments respond sensitively to greenhouse warming. In particular the El Niño-Southern Oscillation (ENSO) cycle obtains more energy, and a tendency toward cold events can be observed. These statistical changes are superimposed on an overall warming trend.

It is suggested that this information can be used in order to refine climate change detection via the optimal fingerprinting strategy. An optimal spectral fingerprint is developed on the basis of linear perturbation theory of wavelet variances. In order to elucidate the potential of higher-order statistical moments in the climate change detection context the optimal spectral fingerprint technique is applied to the ECHAM4/OPYC greenhouse warming simulation. The results provide a rough estimate of the timescale over which human-caused changes in the statistics of ENSO can be expected to exceed the level of natural variability. These results reveal in particular that recent observed changes of ENSO variability are consistent with the null hypothesis of natural climate variability.

Furthermore, an information theoretical approach is adopted to investigate possible influences of global warming on ENSO predictability.
\end{abstract}

\section{Introduction}

Anthropogenic greenhouse gas emissions have increased continuously since the beginning of the industrial revolution. It became scientific consensus (IPCC 1996) that further enhancement of greenhouse gas emissions will oppose humankind with a drastic global climate change. Therefore, it is an urgent demand to unequivocally identify the climate signature of human fossil fuel burning on climate- the so-called anthropogenic fingerprint. In order to guide political decisions controlling future fossil fuel burning it is necessary to monitor how much past greenhouse gas emissions have already contributed to global climate change.

Recent studies (Hegerl et al. 1996; Hegerl and North 1997) using the optimal fingerprint technique (Hasselmann 1993) have revealed that the detection of the anthropogenic fingerprint in the present climate record is just emerging. But a serious caveat in detection studies (Bell 1982, 1986; Barnett 1986; Hasselmann 1993; Santer et al. 1993; North and Kim 1995a,b) is the estimation of the natural variability level, in particular of long-term climate variability either by using observations or by adopting climate model integrations. Furthermore, detection studies have not yet explicitly considered the

Corresponding author address: Dr. A. Timmermann, KNMI, Postbus 201, 3730 AE De Bilt, the Netherlands.

E-mail: timmera@knmi.nl effect of possible interactions between the changing climate mean state and the higher-order statistical moments such as the variance and the skewness.

In a recent study by Palmer (1997) a nonlinear perspective on climate prediction and climate variability is formulated. The idea at the core of this investigation is that the climate attractor can be described by quasistationary regimes. The probability for the climate attractor to stay in one of those regimes is strongly dependent on the presence of external forcing factors such as anthropogenic greenhouse gas forcing. Palmer (1997) investigates the sensitivity of climate variability and the potential for climate forecasts in the presence of an external forcing.

In our study a mathematical framework is formulated in which changes of climate variability can be investigated. Section 2 shall introduce the reader to problems of nonstationary climate statistics. It is demonstrated on the basis of CGCM experiments that the climate sensitivity of higher-order statistical moments is an important feature that has to be taken into account in climate diagnostic studies. Section 3 describes the mathematical framework of the optimal spectral fingerprint, which enables the monitoring of changes of variability in certain frequency bands. In addition, we give a brief review of the optimal fingerprint technique. In order to estimate the possibility of detecting changes of El NiñoSouthern Oscillation (ENSO) statistics, the optimal spectral fingerprinting technique is applied to observations and to the CGCM control and greenhouse gas 
simulations. The results are discussed in section 4. Section 5 investigates the question of whether global warming is capable of changing the characteristics of ENSO predictability. A general information theoretical approach is formulated to account for the dynamics of climate predictability. This paper concludes with a brief discussion and summary of our main findings (section $6)$.

\section{Climate statistics in the tropical Pacific region}

In this section we illustrate that higher-order statistical moments of the tropical climate system, namely, of the El Niño-Southern Oscillation phenomenon, can be rather sensitive to anthropogenic climate change.

The strongest natural interannual climate fluctuation is the ENSO phenomenon, which occurs irregularly with a period of about $2-8$ yr. It can be regarded as a joint atmosphere-ocean oscillation originating in the tropical Pacific. The swings of the ENSO cycle change not only the atmospheric conditions in the Tropics (typical impacts are droughts in Indonesia and northeast Brazil, anomalously wet conditions over Ecuador and Peru, etc.) but also the position of the subtropical jet streams, thereby also encompassing the Northern and Southern Hemispheric climate conditions. The complex and sensitive marine ecological system of the upwelling region near Peru and Ecuador has to be quite flexible in order to adjust to strong climate variations within a few years. The same is true for the socioeconomic systems of those countries that are strongly affected by ENSO. Thus, it becomes an important question as to whether the statistics of interannual climate variability is going to change under greenhouse warming conditions. Can we expect critical ENSO impact regions to suffer from more or less extreme situations in the future? Is there already evidence for a changed level of climate variability in observations? These are questions of great importance that we aim to address below. Furthermore, dramatic changes of climate variability have to be taken into account in the climate detection methodology as discussed by Bell (1986). To put it the other way round, changes of higher-order statistical moments of the climate system can be regarded as part of the greenhouse warming signal and should be considered as an important contribution for detecting human-caused climate change.

In order to study the greenhouse warming impact on ENSO we analyze a control (present-day atmospheric greenhouse concentrations) and a climate change experiment conducted with the Max-Planck Institut coupled atmosphere/isopycnal ocean model (ECHAM4/ OPYC3). The atmosphere model ECHAM4 is coupled to the isopycnal ocean model OPYC3, adopting a soft flux correction method (Bacher et al. 1997). The climate change simulation follows the prescribed greenhouse gas emission scenario IS92a (IPCC 1992) for the $\mathrm{CO}_{2}$ emissions only. In two recent investigations by Roeckner et al. (1996) and Bacher et al. (1997) the ENSO performance of the control integration is validated. It emerges in their studies that the sea surface temperature anomalies (SSTA) related to the El Niño phenomenon have a realistic amplitude and show the typical irregularity of the ENSO signal. The corresponding correlation (teleconnection) patterns of the winds, pressure, and precipitation are very similar to those found in observations. However, the simulated ENSO cycle is too short (period of about $2 \mathrm{yr}$ ) as compared to observations.

Using these CGCM experiments Timmermann et al. (1999) discuss the characteristics of the simulated ENSO response to greenhouse warming. Their findings can be summarized as follows: greenhouse warming changes the statistics of ENSO. Superimposed on an overall warming trend that tends to reduce the zonal equatorial SST gradient, ENSO variability increases and a tendency toward stronger "cold" events becomes apparent. Cold here denotes a cold event relative to a warmer climate mean state. Furthermore, their study suggests that abrupt changes between different climate variability regimes take place. The ENSO period of 2 yr remains relatively stable during the greenhouse warming simulation. Overall, their findings are consistent with a nonlinear system perspective. This analysis shall be continued here focusing more on the statistical aspects of climate change in the tropical Pacific, rather than on the associated physics.

Figure 1 displays the two dominant empirical orthogonal functions (EOFs) of annually averaged surface temperature anomalies ${ }^{1}$ in the Tropics for the control integration, accounting for $35 \%$ and $16 \%$ of the total data variance, respectively. The first EOF shows the typical El Niño structure with strong warming in the eastern equatorial Pacific, whereas the second pattern is associated with ocean cooling and land warming in the Tropics.

These EOF patterns can be chosen as an orthogonal phase space basis. We calculate the projections of monthly control and scenario run SST anomalies onto the EOF patterns shown in Fig. 1. Two-dimensional frequency distributions are estimated to obtain the individual phase space densities (Fig. 2). The phase space ellipsoid of the control integration (Fig. 2, upper panel) characterizes the alternation of ENSO and the tropical cold-ocean warm-land pattern (Fig. 1, lower panel). The ENSO phase space portrait of the greenhouse warming scenario exhibits an overall warming trend, which is represented by the long $40^{\circ}$ tail of the frequency distribution. Furthermore, one observes with increasing values of the projections, and hence with increasing time, a slight broadening of the tail. One way to characterize this behavior is to introduce a skewness parameter that measures the deformation of the frequency distribution along an axis perpendicular to the main trend

\footnotetext{
${ }^{1}$ SSTA over the oceans and 2-m temperature anomalies over land.
} 

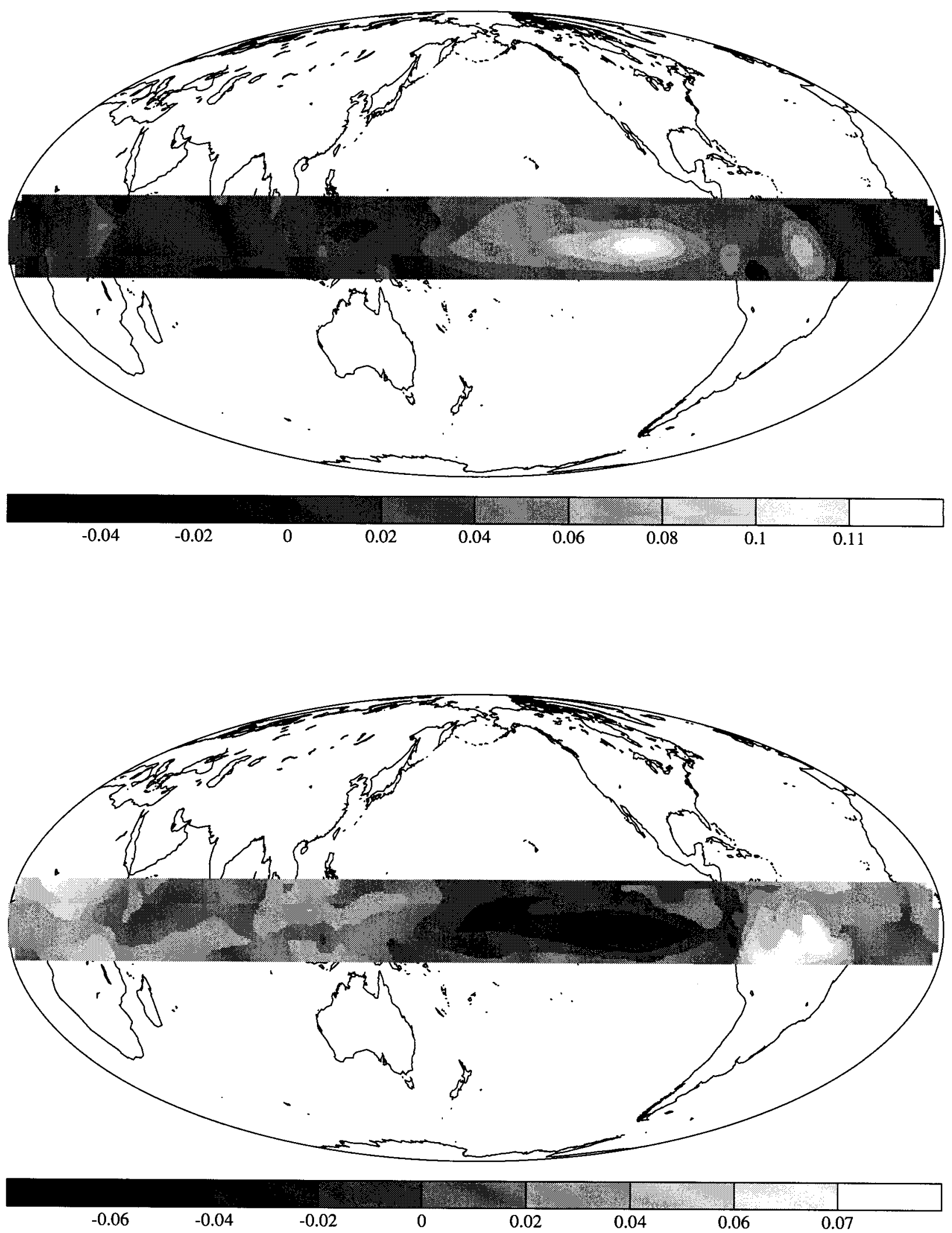

FIG. 1. First (upper) and second (lower) EOF patterns obtained from the annual mean surface temperature anomalies simulated by the ECHAM4/OPYC control integration. 


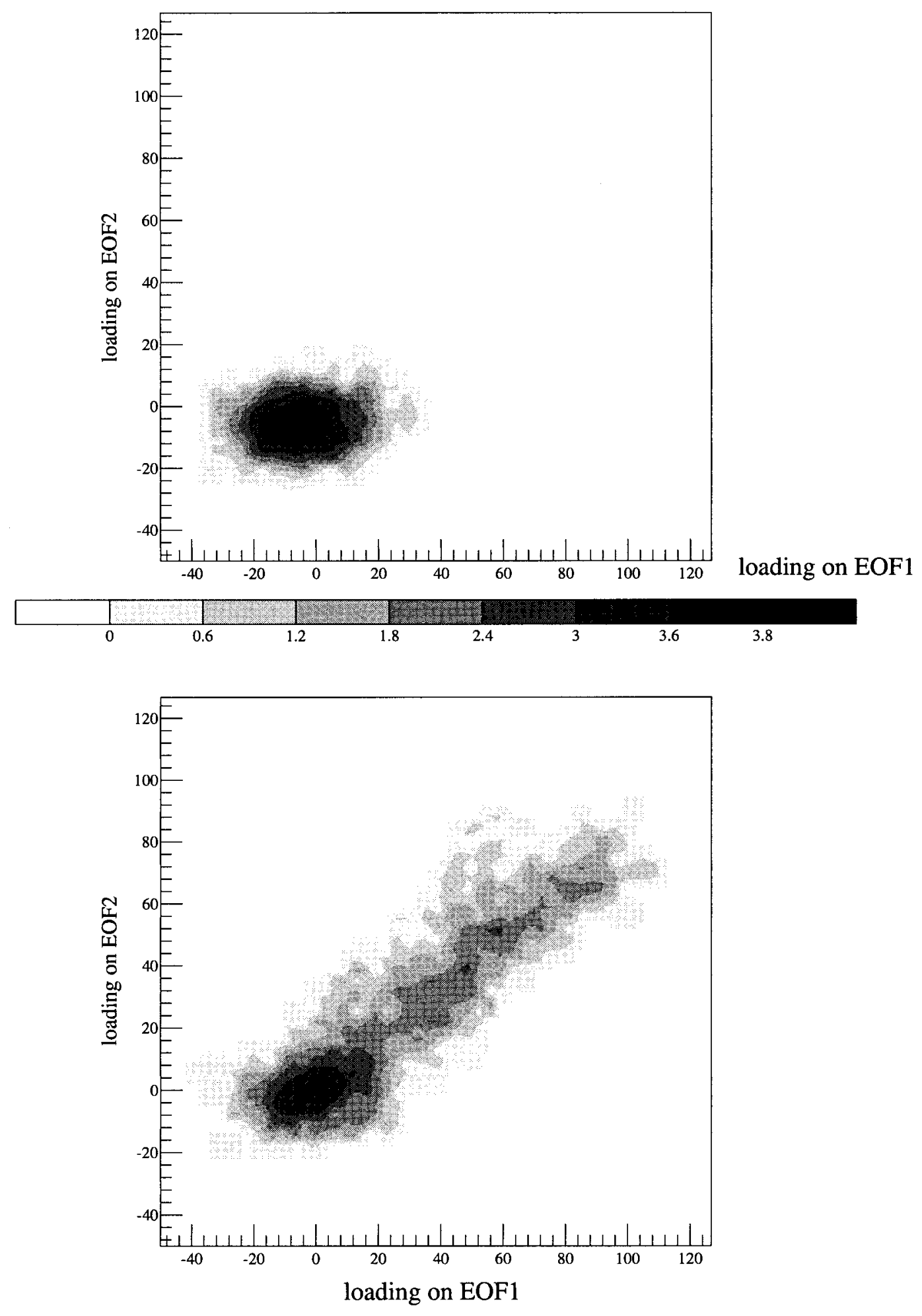

FIG. 2. Phase space portrait of the projections of monthly SST anomalies onto the EOF patterns of Fig. 1. Logarithmic frequency distributions $(+1)$ of the individual projections are displayed for the control integration (upper) and the scenario-A experiment (lower). The $x$ axis represents the loading on EOF1 and the $y$ axis the loading of the anomalies on EOF2. 
axis. Another interpretation could be that the observed diffusion of probability as a function of time is the expression of a bimodal structure. In this case a secondary trend trajectory lying off the major trend direction introduces a bimodal splitting, an increase of variance, and changes of the skewness as a function of the control parameter. A slight indication for such a splitting and for a secondary trend component can be seen in Fig. 2 (lower panel).

As already mentioned, the broadening of the scenario phase space trajectory of SST anomalies leads to an increase of variance and is also characterized by a slight asymmetry, leading to a change of the skewness. Skewness is defined as the normalized third statistical moment (see, e.g., Nakamura and Wallace 1991). Here we calculate this quantity at each grid point for the simulated scenario surface temperature anomaly and sea level pressure anomaly (SLPA) fields. Figure 3 depicts the skewness parameter field as obtained from the monthly SSTA field of the first 30 integration years (1860-1890, upper panel) and the last 30 integration years (20702100 , lower panel) of the global warming scenario (after removing the quadratic trend). In order to assign statistical significance to the skewness parameter we use the approximation formula for the $95 \%$ significance value of the skewness: $S_{95 \%}=2 \sqrt{6 / N}$, where $N$ represents the number of independent samples (White 1980). In our case we analyze 360 months. Typical SST anomalies have decorrelation times of a few (1-4) months. Thus we end up with a significance level of not more than 0.5 . Let us therefore interpret only changes in the skewness parameter that exceed this value. It emerges from Fig. 3 that for a rather large region between $20^{\circ} \mathrm{S}$ and $20^{\circ} \mathrm{N}$ the frequency distributions of the SSTA are bent out of shape toward a preference of cold events. Since the dynamics of SST anomalies in the tropical Pacific are strongly related to changes in the atmospheric variables and vice versa, we expect that also the atmospheric variables such as SLP exhibit a shift toward nonsymmetric frequency distributions. We calculate the skewness parameter field for the SLP anomalies from our scenario-A integration in the same manner as described for the SSTA. The result for the first 30 integration years is displayed in the upper panel of Fig. 4 and the result for the last 30 years of the greenhouse simulation is depicted in the lower panel. Since the decorrelation time of tropical atmospheric anomalies is smaller than for oceanic variables (on the order of 1-2 months) we obtain skewness values at the $95 \%$ significance level of about 0.35 . Figure 4 illustrates that large changes of the skewness parameter for the SLP anomalies occur in the east and west equatorial Pacific. This is an expression of the fact that due to the strong atmosphere-ocean feedbacks in the Tropics a tendency toward stronger cold events is associated with a tendency toward stronger trade wind anomalies.

Skewness values significantly different from zero might be regarded as a signature of an underlying non- linearity. The indication of a bimodal phase space trajectory and the temporal evolution of the skewness for surface temperature and SLP anomalies in the Tropics toward more asymmetry, both indicate that the dynamics of El Niño-related climate variability is subject to nonlinear interactions.

\section{The optimal spectral fingerprint}

In this section we intend to combine wavelet analysis with the optimal fingerprint method. Crudely speaking, a wavelet decomposition is an expansion of an arbitrary function into smooth localized contributions labeled by a scale and a position parameter. Wavelet analysis is a good tool to resolve spectral characteristics as a function of time. In contrast to Fourier analysis with a sliding window, wavelet analysis does not suffer from the stationarity assumption (Kumar and Foufoula-Georgiou 1994). Wavelets resolve the phase space of time series in an optimal way (Kumar and Foufoula-Georgiou 1994). Let us consider the climate variable $x_{i}(t)$ (e.g., the vector time series of the SST anomalies). ${ }^{2}$ This variable shall be particularly sensitive to the anticipated climate change expected from a global warming scenario. It can be decomposed into its spectral contributions as a function of time using the wavelet decomposition:

$$
w_{i}(a, t)=\int_{-\infty}^{\infty} \psi^{*}\left(\frac{t-t^{\prime}}{a}\right) x_{i}(t) d t^{\prime},
$$

where $a$ denotes the timescale and $\psi^{*}$ the complex conjugate of the so-called mother wavelet $\psi$. Wavelets as considered in this context belong to the quadratic Lebesgue integrable functions on a compact support. We do not want to go into mathematical details here and refer the reader to standard textbooks on wavelets. The family of wavelets is obtained by expanding and translating the mother wavelet by $a$ and $t^{\prime}$, respectively. In order to obtain the total wavelet variance of the signal in the spectral range $A=\left[a_{1}, a_{2}\right]$ we calculate the expression

$$
e_{i}^{A}(t)=\int_{a_{1}}^{a_{2}}\left|w_{i}(a, t)\right|^{2} \frac{d a}{a^{2}} .
$$

The spectral range index $A=\left[a_{1}, a_{2}\right]$ can be chosen either on account of some a priori information or in an appropriate manner, for example, by maximizing the climate signal to noise ratio.

The following part is a brief outline of the detection strategy of Hasselmann (1993), which instead of being applied to the normal climate signal $x_{i}(t)$ is applied to the corresponding climate variability signal $e_{i}^{A}(t)$. Let us consider the case $A=\left[a_{1}, a_{2}\right]$ being fixed due to some

\footnotetext{
${ }^{2}$ The index of $x_{i}(t)$ represents the site number $i$ in a discretized space (grid variables).
} 

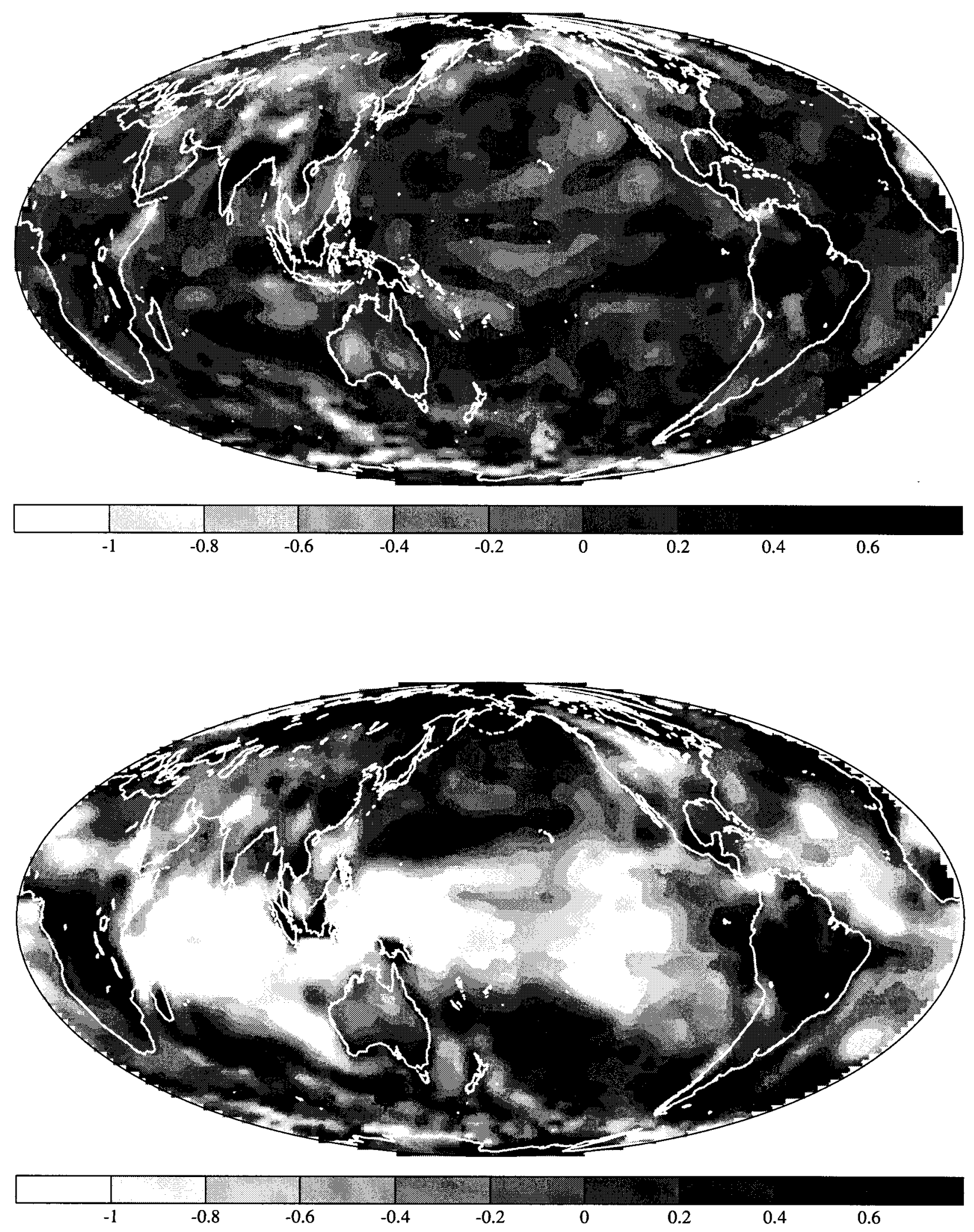

FIG. 3. Skewness parameter of surface temperature anomalies obtained from the first (upper) and last (lower) 30 years of the scenario-A experiment. Prior to the analysis all data were detrended quadratically. 

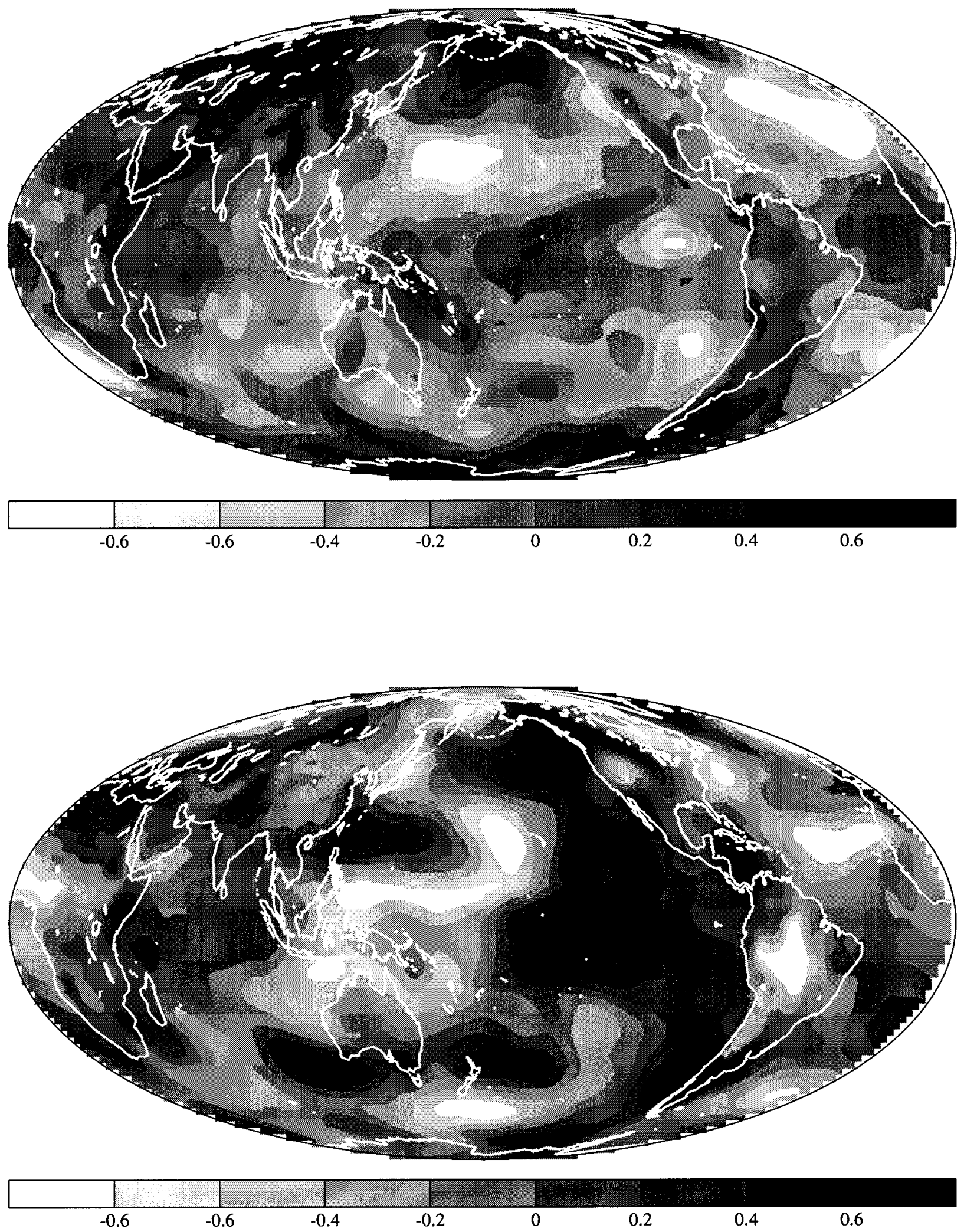

FIG. 4. Skewness parameter of sea level pressure anomalies obtained from the first (upper) and last (lower) 30 years of the scenario-A experiment. Prior to the analysis all data were detrended quadratically. 
a priori information. For this case we omit the spectral range index. In a climate change scenario we make the following linear separation expression for the vector time series of the wavelet energy

$$
e_{i}(t)=e_{i}^{s}(t)+\tilde{e}_{i}(t)
$$

where $e_{i}^{s}(t)$ is the climate change component (the signal), which we plan to detect; and $\tilde{e}_{i}(t)$ represents the natural variability component. In order to obtain a compact tensor notation ${ }^{3}$ the covariance tensor of natural climate variability

$$
C_{i j}=\mathcal{E}\left[\tilde{e}_{i}(t) \tilde{e}_{j}(t)\right]
$$

is chosen as an appropriate metric. Here $\mathcal{E}$ denotes the ensemble mean. A first guess of $e_{i}$ denoted by $s_{i}$ can be estimated, for example, as the leading EOF of $e_{i}(t)$ obtained from a CGCM global warming simulation. Rotating $s_{i}$ away from the regions of high variance in $\tilde{e}_{i}(t)$ by the so-called optimal fingerprint

$$
\sigma_{i}=C_{i j}^{-1} s^{j}
$$

yields an optimal squared signal to noise ratio,

$$
R^{2}=\frac{\left(\sigma^{i} e_{i}^{o b s}\right)^{2}}{\mathcal{E}\left[\left(\sigma^{i} \tilde{e}_{i}\right)^{2}\right]},
$$

where $e_{i}^{\text {obs }}$ is obtained from (1) and (2) by taking observational data. Let us recall that $e_{i}(t)$ is a function of the spectral range $A=\left[a_{1}, a_{2}\right]$. By changing $A$ it is possible to optimize the signal to noise ratio $R$ even further. One obtains the condition

$$
\frac{\partial}{\partial a_{j}}\left(\frac{\left(\sigma^{i} e_{i}^{A, o b s}\right)^{2}}{\mathcal{E}\left[\left(\sigma^{i} \tilde{e}_{i}^{A}\right)^{2}\right]}\right)=0, \quad j=1,2 .
$$

The projections of the observations and the natural variability data (estimated, e.g., from a control simulation of a CGCM) on the optimal fingerprint $\sigma_{i}$-the so-called detection variables - are subject to statistical tests. Detection at a prescribed significance level is achieved when the hypothesis that the observed detection variable can be explained by natural climate variability alone is rejected at that statistical level.

\section{Optimal wavelet fingerprinting of ENSO}

In order to apply the optimal spectral fingerprint to the simulated ENSO behavior it appears suitable (Hegerl et al. 1997) to reduce the dimensionality of the problem. Concentrating only on the dominant EOF modes yields

\footnotetext{
${ }^{3}$ Covariant and contravariant tensor notation shall be used. The summation convention over same indices is used. A covariant metric tensor $g_{i k}$ is introduced to account for the geometry of the problem. Using the following relation $g_{i j} g^{j k}=\delta_{i k}$ (Kronecker symbol) it is possible to switch from covariant to contravariant indices and vice versa by means of the following equations $X \ldots i \ldots=\mathrm{g}^{i j} X_{\ldots j \ldots}$ and $X \ldots i \ldots=\mathrm{g}_{\mathrm{ij}} X \ldots j \ldots$
}

an appropriate representation. Here we calculated the 10 leading control run EOFs of the interannual (2-5 yr) wavelet energy of tropical SST anomalies. ${ }^{4}$ Continental grid points are masked. Figure 5 depicts the three leading EOF modes of natural variability of the computed interannual wavelet energy. The pattern of the first EOF mode implies that also the dominant variability of interannual climate fluctuations in the Tropics is associated with the typical El Niño-like structure. The second and third EOFs accounting for considerably less variance are characterized by smaller spatial structures, which are also mainly concentrated in the east Pacific. In this ten-dimensional EOF basis the estimated covariance matrix of "natural" model variability obtains a simple diagonal form. It should be noted here that due to the shortness of the observations the natural covariance tensor is estimated from the CGCM control run; hence, our results have to be interpreted in this model world of natural variability. A successful detection of human-caused climate patterns is based on the estimation of a first guess of the expected climate change signal. Here the guess pattern is estimated from the leading EOF mode of interannual wavelet energy as simulated by the greenhouse warming experiment. The optimal fingerprint is then obtained by rotating this guess pattern into those directions that are less contaminated by natural noise. A detection variable can then be calculated by projecting the data onto the optimal fingerprint.

In order to determine the relative contribution of the different EOF patterns to the rotation angles in the tendimensional EOF space, we calculate the projection of the signal guess pattern onto the different control run EOFs, weighted by the inverse of the respective eigenvalues. These contributions can also be interpreted as the individual EOF contributions to the squared signal to noise ratio. Figure 6 shows the result for the 10 leading EOFs. One observes that the third EOF (shown in Fig. 5, lower panel) contributes strongest to the signal to noise ratio. The contribution of higher-order EOFs (4-10) is quite small. A detection problem can only be solved adequately if the relative role of higher-order EOF patterns, which are characterized by small spatial scales and which might get unphysical and arbitrary with respect to prior data filtering and the choice of the geographical region, decreases rapidly. This implies that the projection of the signal onto the noise EOFs has to shrink much faster than the associated EOF eigenvalues. Obviously this is the case in our analysis. Figure 7 displays the estimated guess pattern and the optimal fingerprint as computed from the signal to noise maxi-

\footnotetext{
${ }^{4}$ The wavelet energy is calculated at each grid point by using the Morlet wavelet. In order to avoid energy leakage the time series is buffered on both ends by a fitted autoregressive process of the second order, which yields rather stable wavelet spectra for the full time range.
} 

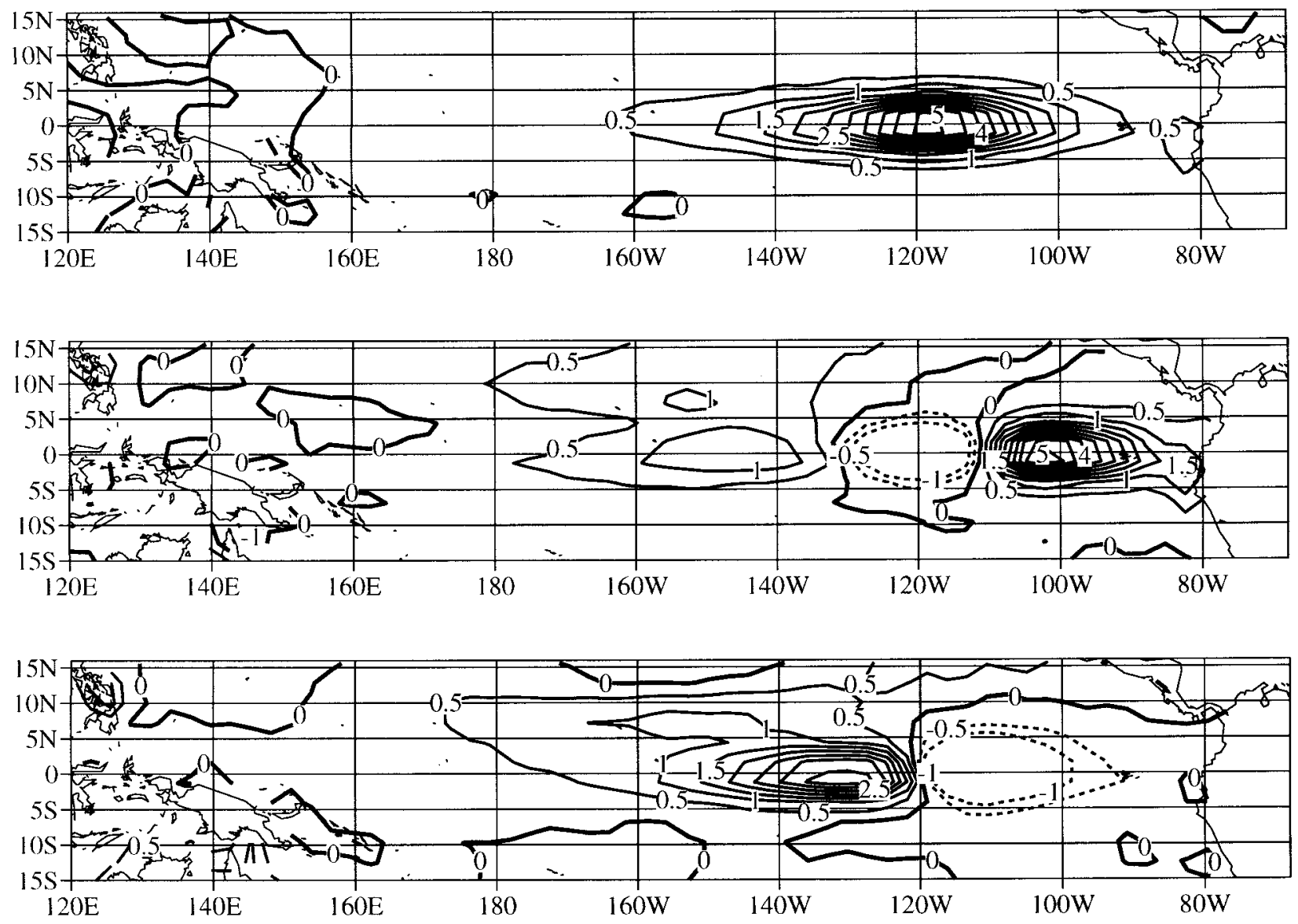

FIG. 5. (upper) First EOF of the interannual SSTA wavelet energy as obtained from the control experiment accounting for $78 \%$ of the variance; (middle) second EOF (8\%); (lower) third EOF (5\%).

mizing rotation in the ten-dimensional EOF space. The guess pattern reveals that the ENSO response to greenhouse warming is to first-order parallel to the dominant variability pattern. This strongly justifies the application

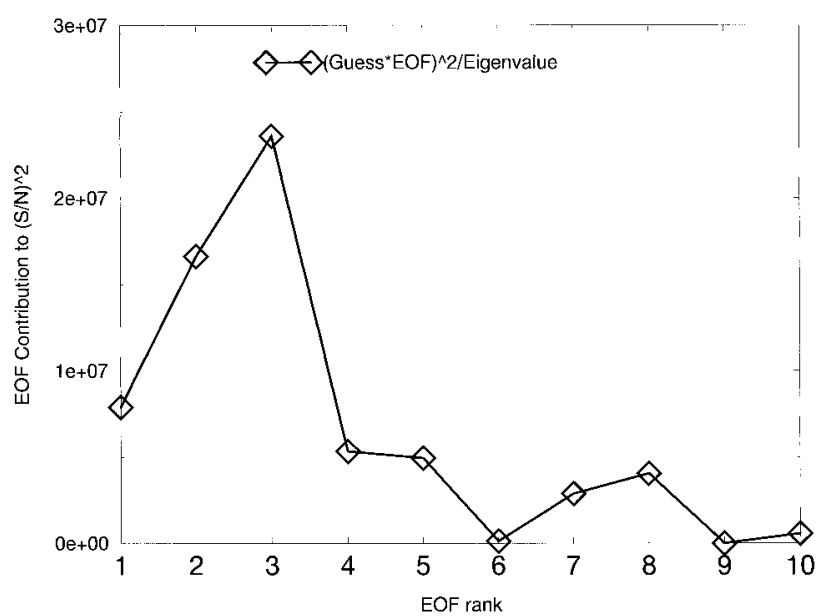

FIG. 6. Contribution of the natural noise EOFs (Fig. 5) to the squared signal to noise ratio. of an optimized detection approach. The optimal fingerprint of interannual wavelet energies is characterized by smaller spatial structures, originating mainly from the contributions of the second and third control run EOFs (see Fig. 5).

In order to obtain the detection variable, being at the core of the detection problem, we project the interannual SSTA wavelet energies from the observations [Global Sea-Ice and SST (GISST) dataset 2.2 from 1903 to 1994, obtained from the Hadley Centre, United Kingdom], the control and scenario experiments onto the optimal fingerprint. The resulting detection variable of the control simulation yields the confidence levels of natural variability. Here we aim to explore whether the observed ENSO evolution deviates substantially from the null hypothesis of natural variability. Figure 8 shows the temporal evolution of the detection variable for the interannual wavelet energies of the greenhouse warming scenario and the observations. In order to allow for a quantitative statistical comparison of the detection variables, a \pm 1 and 2 standard deviation confidence tube of natural control run variability is also depicted. It should be noted here that the wavelet energy represents somehow the 

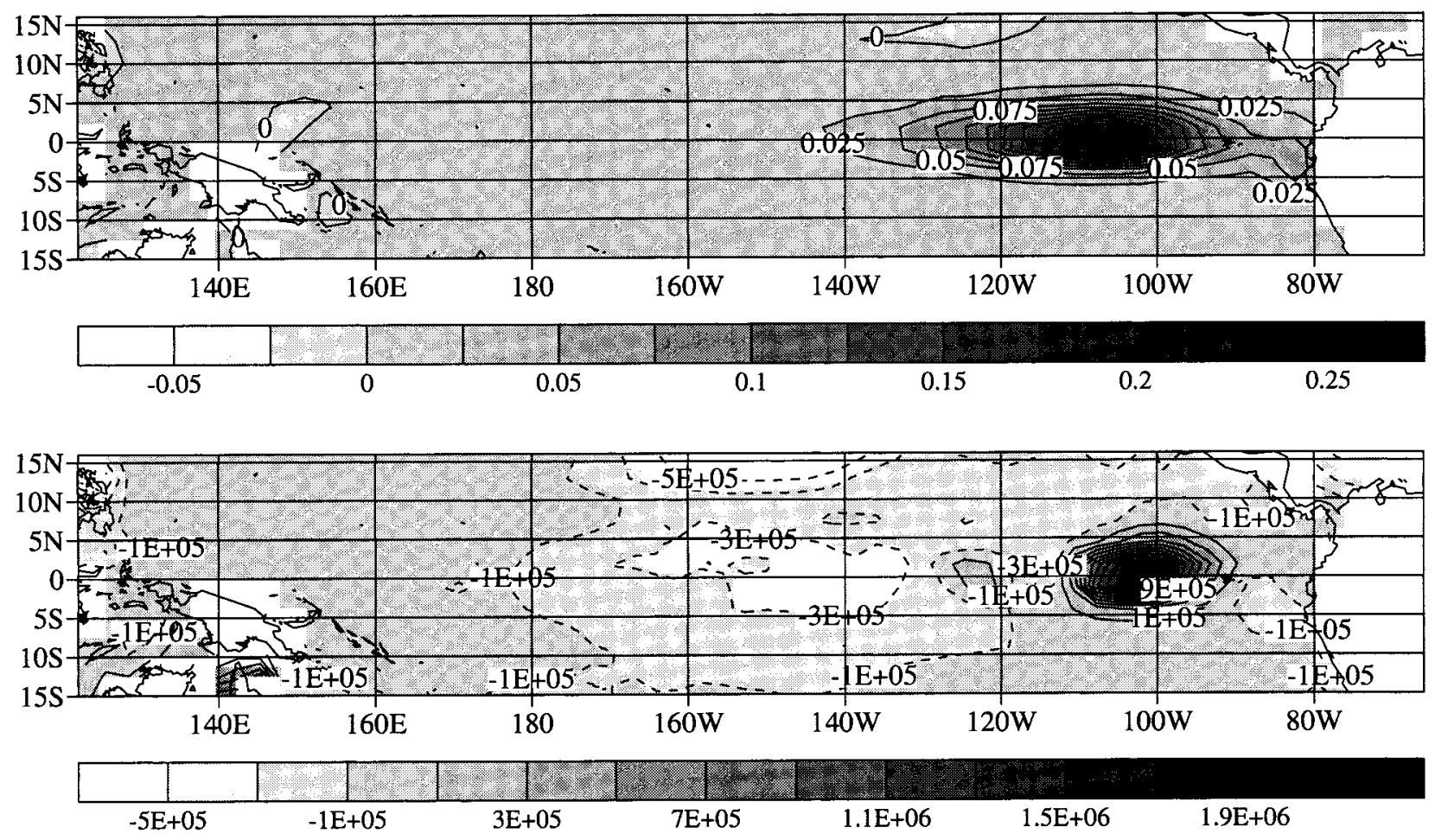

FIG. 7. (upper) Guess pattern of the interannual SSTA wavelet energy as obtained from the first EOF of the scenario-A experiment; (lower) optimal spectral fingerprint as obtained from the signal to noise maximizing rotation of the guess pattern in the ten-dimensional wavelet energy EOF space of the control run.

weighted sum of squares of approximately Gaussiandistributed random variables. The corresponding distribution of this sum is a $\chi^{2}$ distribution. However, estimating the corresponding number of degrees of freedom is somehow ambiguous in our situation. Figure 8 reveals that the observed time evolution of ENSO variability is consistent with the null hypothesis of natural variability. The greenhouse warming experiment shows a very strong ENSO sensitivity and the detection variable leaves the confidence limit around the model year 2000. However, a reliable estimation of the time when the scenario run leaves the confidence limit has to take into

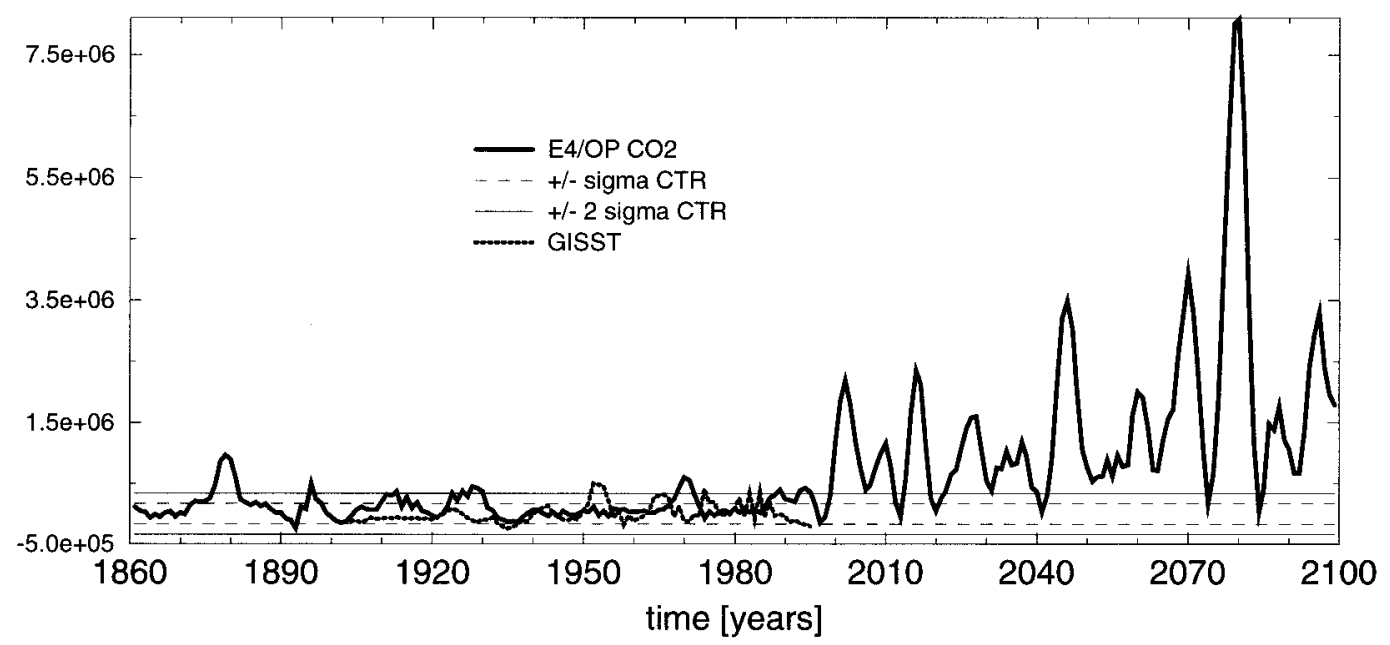

FIG. 8. Detection variable of the interannual SSTA wavelet energy (2-5 yr) as obtained from the projection of the wavelet energy onto the optimal spectral fingerprint (see Fig. 7, lower panel). The detection variables are depicted for the GISST dataset and the scenario-A integration of ECHAM4/OPYC3. Two confidence limits are shown that characterize the natural variability of the wavelet energies as simulated by the control run. 
account the fact that the sensitivity of different global warming trajectories has a statistical spread.

\section{Climate predictability and anthropogenic greenhouse warming}

Apart from just understanding the long-term behavior and sensitivity of interannual climate variability it is also interesting to study climate, and in particular ENSO, predictability under changed climate conditions. For this purpose we introduce a general information theoretical functional (Gelfand and Yaglom 1957; Leung and North 1990; Palus 1993). The basic idea is to define a kind of moving nonlinear autocorrelation for a time series quantifying the local amount of information that is contained in the variable $x$ at time $t$ about the variable $x$ at time $t+\tau$. Some further illustration about this method is given below. Let $X$ be a random variable with a set of values $\chi$ and a probability density $p(x), x \in \chi$. The entropy $H(X)$ of a random variable $X$ is defined as

$$
H(X)=-\sum_{x \in X} p(x) \ln p(x) .
$$

For a pair of random variables $X, Y$ with a joint probability density $p(x, y)$ the joint entropy $H(X, Y)$ is given by

$$
H(X, Y)=-\sum_{x \in X} \sum_{y \in \psi} p(x, y) \ln p(x, y) .
$$

The average amount of information contained in the common dynamics of the variables $X$ and $Y$ is quantified by the mutual information $I(X, Y)$, which is defined as

$$
I(X, Y)=H(X)+H(Y)-H(X, Y) .
$$

The idea that links this general nonlinear concept to predictability is to quantify the amount of information contained in the variable $X(t)$ about the variable $X(t+$ $\tau)$, also sometimes referred to as the redundancy of $X(t)$ and $X(t+\tau)$. In this sense the mutual information can be regarded as a kind of nonlinear correlation for the prediction horizon $\tau$. In this study we compute the redundancy from the data under consideration and a set of surrogate data, which shall be seen as a representation of a null hypothesis model. We analyze the statistical quantity that is computed from the absolute value of the difference between the redundancy value obtained from the data and a mean value of a set of surrogates in standard deviations of the latter. The predictability $I(x(t)$, $x(t+\tau))$ and the related statistics are evaluated in sliding overlapping windows with a length of $N_{w}$ samples and a window step of $N_{s}$ samples. Our working hypothesis is stepwise stationarity and ergodicity for the time series under study. The resulting relative redundancy is expressed in standard deviations of the surrogate redundancy and as a function of model time. This technique is applied to the Niño 3 SSTA time series of the ECHAM4/OPYC3 control and scenario-A integration (upper panel of Fig. 9; the quadratic trend of the
scenario-A time series is not removed). The time series of the nonlinear relative redundancies are tested against white noise surrogates. We use a window length of $N_{w}$ $=256$ samples (months), a window step of $N_{s}=3$ samples, and a prediction horizon of $\tau=6$ months. The predictability measures are shown in Fig. 9 (lower panel). Apparently, there is no clear statistical deviation of the predictability measure in the greenhouse warming scenario as compared to the control run. But an interesting feature is the interdecadal modulation of El Niño predictability. Whether this is an expression of the stochastic nature of ENSO or whether it is related to welldetermined oscillatory physical processes has to be clarified further. Apart from this univariate example, interesting information can be obtained also from the dominant patterns of multivariate information theoretical functionals.

\section{Conclusions}

Recently, some intense debate started on the question whether ENSO statistics has already changed significantly during the last few decades (Enfield and Cid 1991; Trenberth and Hoar 1996, 1997; Rajagopolan et al. 1997). "Unusual" behavior, such as the long warming period in the tropical Pacific during the early 1990s or an intensification of ENSO variability since the 1970s (Gu and Philander 1995) has to be tested against statistical null hypothesis models for ENSO variability, which are formulated mostly in terms of linear stationary stochastic processes. Detecting nonstationarity of ENSO, which can either be due to internal variability or due to external forcing factors, can be facilitated by taking into account ENSO statistics, which is simulated by realistic CGCMs. This avoids the somewhat arbitrary adoption of explicit stochastic null hypothesis models.

Our study aimed to exploit such CGCM information in order to detect changes in the ENSO variance. It was documented by a control and greenhouse warming simulation conducted with a realistic CGCM that higherorder statistical moments of climate variables carry important information on the greenhouse warming signal and may thus improve recent estimates of climate change detection. An optimal spectral fingerprint was developed that allows for the detection of changes of climate variability. Applying this technique to the interannual SST variability in the tropical Pacific provides a rough estimate of the timescale over which humancaused changes in the ENSO statistics can be expected to exceed the level of natural variability. Our results reveal in particular that recent observed changes of ENSO variability are consistent with the null hypothesis of natural climate variability.

Furthermore, the issue was raised as to whether the general predictability characteristics of ENSO are subject to global warming. Using an information theoretical approach no significant change of ENSO predictability 

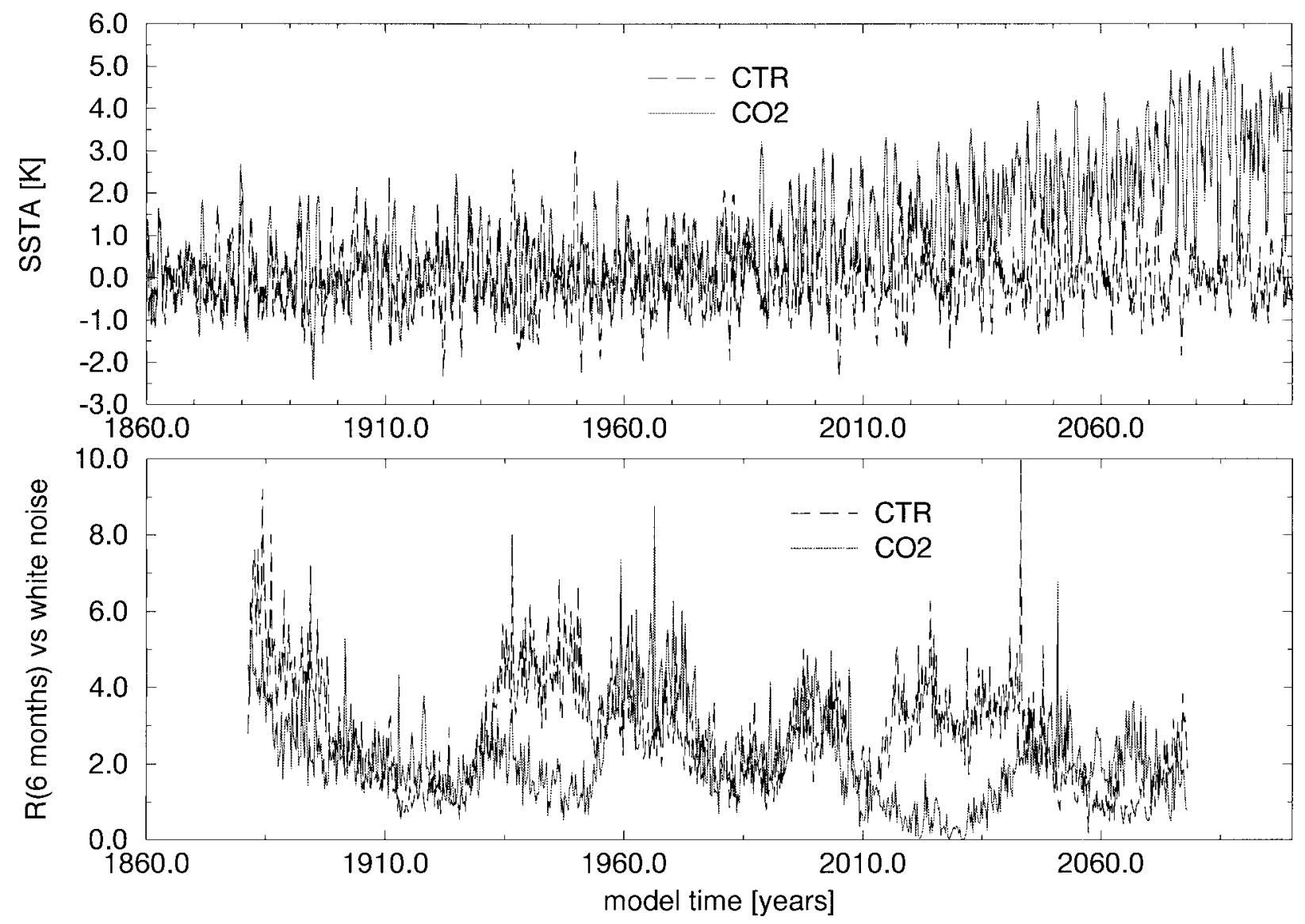

FIG. 9. (upper) Niño 3 SSTA time series for the ECHAM4/OPYC control and the transient greenhouse warming experiment; (lower) Relative redundancies for the Niño 3 SST anomalies for a prediction horizon of 6 months. The corresponding surrogate data simulate a white noise process.

could be detected, which might have been due to nonlinearities and changes of the ENSO irregularity.

Acknowledgments. This work was partly supported by the German government under Grant 07VKV01/1 and the EU project ENV4-CT98-0714 (SINTEX). I gratefully acknowledge the helpful comments and suggestions of Profs. K. Hasselmann and G. North. Thanks also to Drs. P. Heimbach, M. Latif, D. Müller, G. Hegerl, M. Allen, and G. Lohmann for fruitful discussions. The CGCM data were kindly provided by Drs. A. Bacher, J. M. Oberhuber, E. Roeckner, and M. Esch.

\section{REFERENCES}

Bacher, A., J. M. Oberhuber, and E. Roeckner, 1997: ENSO dynamics and seasonal cycle in the tropical Pacific as simulated by the ECHAM4/OPYC3 coupled general circulation model. Climate Dyn., 14, 431-450.

Barnett, T. P., 1986: Detection of changes in global tropospheric temperature field induced by greenhouse gases. J. Geophys. Res., 91, 6659-6667.

Bell, T. L., 1982: Optimal weighting of data to detect climatic change:
Application to the carbon dioxide problem. J. Geophys. Res., 87, 11 161-11 170.

_ 1986: Theory of optimal weighting of data to detect climatic change. J. Atmos. Sci., 43, 1694-1710.

Enfield, D. B., and L. S. Cid, 1991: Low-frequency changes in El Niño-Southern Oscillation. J. Climate, 4, 1137-1146.

Gelfand, I. M., and A. M. Yaglom, 1957: Computing information about a random function that is contained in another one (in Russian). Sov. Phys.-Uspekhi, 12 (1), 3-52.

Gu, D., and S. G. H. Philander, 1995: Secular changes of annual and interannual variability in the Tropics during the past century. $J$. Climate, 8, 864-876.

Hasselmann, K., 1993: Optimal fingerprints for the detection of time dependent climate change. J. Climate, 6, 1957-1971.

Hegerl, G. C., and G. R. North, 1997: Comparison of statistically optimal approaches to detecting anthropogenic climate change. J. Climate, 10, 1125-1133.

- H. von Storch, K. Hasselmann, B. D. Santer, U. Cubasch, and P. D. Jones, 1996: Detecting greenhouse gas induced climate change with an optimal fingerprint method. J. Climate, 9, 22812306.

IPCC, 1992: Climate Change 1992: The Supplementary Report to the IPCC Scientific Assessment. J. T. Houghton et al., Eds., Cambridge University Press, $200 \mathrm{pp}$.

- 1996: Climate Change 1996: The IPCC Second Scientific Assessment. J. T. Houghton and M. Filho, Eds., Cambridge University Press, $572 \mathrm{pp}$. 
Kumar, P., and E. Foufoula-Georgiou, 1994: Wavelet analysis in geophysics: An introduction. Wavelets in Geophysics, E. FoufoulaGeorgiou and P. Kumar, Eds., Academic Press, 1-43.

Leung, L.-Y., and G. R. North, 1990: Information theory and climate prediction. J. Climate, 3, 5-14.

Nakamura, H., and J. M. Wallace, 1991: Skewness of low-frequency fluctuations in the tropospheric circulations during Northen Hemisphere winter. J. Atmos. Sci., 48, 1441-1448.

North, G. R., and K. Y. Kim, 1995a: Detection of forced climate signals. Part I: Filter theory. J. Climate, 8, 401-408.

, and - 1995b: Detection of forced climate signals. Part II: Simulation results. J. Climate, 8, 409-417.

Palmer, T. N., 1997: A nonlinear dynamical perspective on climate prediction. J. Climate, 12, 575-591.

Palus, M., 1993: Identifying and quantifying chaos by using information-theoretic functionals. Time Series Prediction: Forecasting the Future and Understanding the Past, A. D. Weigend and N. A. Gershenfeld, Eds., Santa Fe Institute Studies in the Sciences of Complexity, Vol. XV, Addison-Wesley, 387-413.
Rajagopolan, B., U. Lall, and M. Cane, 1997: Anomalous ENSO occurences: An alternate view. J. Climate, 10, 2351-2357.

Roeckner, E., J. M. Oberhuber, A. Bacher, M. Christoph, and I. Kirchner, 1996: ENSO variability and atmospheric response in a global coupled atmosphere-ocean GCM. Climate Dyn., 12, 737-754.

Santer, B. D., T. M. L. Wigley, and P. D. Jones, 1993: Correlation methods in fingerprint studies. Climate Dyn., 8, 265-276.

Timmermann, A., J. M. Oberhuber, A. Bacher, M. Esch, M. Latif, and E. Roeckner, 1999: ENSO response to greenhouse warming. Nature, in press.

Trenberth, K., and T. Hoar, 1996: The 1990-1995 El Niño-Southern Oscillation event: Longest on record. Geophys. Res. Lett., 23, 57-60.

- and - 1997: El Niño and climate change. Geophys. Res. Lett., 24, 3057-3060.

White, G. H., 1980: Skewness, kurtosis and extreme values of Northern Hemisphere geopotential heights. Mon. Wea. Rev., 108, 1446-1455. 